\title{
Innovación educativa en el aula mediante Design Thinking y Game Thinking
}

\section{Educational innovation in the classroom through Design Thinking and Game Thinking}

\author{
Hugo Arias-Flores ${ }^{1}$ \\ https://orcid.org/0000-0003-0106-6661 \\ Janio Jadán-Guerrero ${ }^{2}$ \\ http://orcid.org/0000-0002-3616-2074 \\ Lucía Gómez-Luna ${ }^{3}$ \\ https://orcid.org/0000-0001-6068-5206 \\ Universidad Tecnológica Indoamérica, Ecuador
}

Recibido: 19-01-2019

Aceptado: 03-04-2019

\section{Cita Recomendada}

Arias-Flores, H., Jadán-Guerrero, j. \& Gómez-Luna, L. (2019). Innovación Educativa en el aula mediante design thinking y game thinking. Hamut'ay, 6(1), 82-95.

http://dx.doi.org/10.21503/hamu.v6i1.1576

\section{RESUMEN}

La tecnología está cambiando los hábitos de las personas y se está insertando con rapidez en la Educación. Cada vez aparecen nuevos recursos educativos apoyados por la tecnología que fomentan la innovación en el aula. En este contexto los maestros afrontan nuevos retos para cambiar rutinas e inercias pedagógicas tradicionales en aulas dinámicas e interactivas. Estos recursos tecnológicos están revolucionando la manera de enseñar en el aula, no solo en el contenido sino también, en la retención del conocimiento y experiencia del estudiante. Este estudio describe una experiencia de innovación educativa en el aula mediante las metodologías Design Thinking y Game Thinking. El objetivo es introducir el uso de tecnologías disruptivas en el aula mediante estrategias lúdicas y pensamiento de diseño para su aplicación didáctica. El estudio se llevó a cabo en un programa de Maestría de Educación, Innovación y Liderazgo en una universidad de Quito, Ecuador. La muestra fue de 120 maestros de educación primaria, secundaria y superior del curso de Infopedagogía Educativa. Estas metodologías permitieron generar resultados innovadores, no sólo en la aplicación sino también en la generación de novedosos recursos educativos tecnológicos. Estos resultados se clasifican en tres categorías: 1. Herramientas de evaluación y coevaluación como Kahoot, Quuizizz y Plickers que generan motivación en el aula; 2. Recursos educativos con códigos QR y realidad aumentada, donde los marcadores y etiquetas NFC proporcionan información adicional para combinar libros interactivos y juegos de mesa con dispositivos móviles; 3. Recursos educativos tangibles,

\footnotetext{
1 Ingeniero en Marketing y Negociación Comercial Internacional y Maestría en Administración de Empresas y Marketing en la Universidad Tecnológica Indoamérica. Editor asociado de la Revista CienciAmérica, facultad de Ingenierías y Tecnologías de la Información y Comunicación, su línea de investigación es políticas públicas de inserción laboral de personas con discapacidad. E-mail cienciamerica@ uti.edu.ec

2 Doctor en Ciencias de la Computación de la Universidad de Costa Rica. Director de Investigación de la Universidad Tecnológica Indoamérica en Ecuador, su línea de investigación es Interacción Humano-Computador y Tecnologías para la Educación Especial. E-mail janiojadan@uti.edu.ec 3 Licenciada en Restauración de Arte y museología de la Universidad Tecnológica Equinoccial. Analista del Centro de Transferencia y Tecnología. E-mail luciagomez@uti.edu.ec
} 
que integran el dispositivo Makey-Makey y Scratch con materiales tradicionales del aula, tales como frutas, plastilina, papel de aluminio o agua. Como conclusión, se establece que el trabajo en equipo fue una de las características más importantes en el aprendizaje basado en el pensamiento de diseño, que permitió el trabajo en proyectos reales con metodologías de innovación y creatividad..

Palabras Clave: Creatividad, innovación, estrategias lúdicas, pensamiento de diseño, pensamiento de juegos.

\section{Abstract}

Technology is changing the habits of people and it is being inserted quickly in Education. New educational resources supported by technology aimed to encourage innovation in the classroom are increasingly appearing. In this context, teachers face new challenges to change routines and traditional pedagogical habits into dynamic and interactive classrooms. These technological resources are revolutionizing the way of teaching in the classroom, not only in the content but also in the retention of knowledge and experience of the student. This study describes an educational innovation experience in the classroom through the Design Thinking and Game Thinking methodologies. The objective is to introduce the use of disruptive technologies in the classroom through playful strategies and design thinking for its didactic application. The study was carried out in a Master's program in Education, Innovation and Leadership at a university in Quito, Ecuador. The sample was composed of 120 teachers of primary, secondary and higher education of the Educational Infopedagogy course. These methodologies are allowed generating innovative results, not only in the application but also in the generation of new technological educational resources. These results are classified into three categories: 1. Evaluation and co-assessment tools such as Kahoot, Quuizizz and Plickers that generate motivation in the classroom; 2. Educational resources with QR codes and augmented reality, where NFC markers and labels provide additional information to combine interactive books and board games with mobile devices; 3. Tangible educational resources, which integrate the Makey-Makey and Scratch device with traditional classroom materials, such as fruit, plasticine, aluminum foil or water. In conclusion, it is established that teamwork was one of the most important characteristics in learning based on design thinking, which allowed to work on real projects with innovation and creativity methodologies.

Keywords: Creativity, innovation, playful strategies, design thinking, game thinking.

\section{INTRODUCCIÓN}

Hoy en día, el trabajo de la innovación y del cambio digital no es una tarea fácil para muchos maestros. El desconocimiento de la utilidad de la tecnología hace que sea complicado aplicar estrategias innovadoras en el aula. Además, muchos de los proyectos basados en la tecnología tienen un gran enemigo: el tiempo, ya que los maestros necesitan de él para actualizarse, explorar y poner en práctica nuevas estrategias apoyadas con la tecnología (Bautista, Martínez \& Hiracheta, 2014), de ahí nace una pregunta de investigación ¿Cómo fomentar en los maestros el uso y aplicación de tecnologías, para que sus clases sean más dinámicas y participativas?

La innovación educativa no se refiere solo a la tecnología sino también a la pedagogía, la didáctica, las estrategias, los procesos, los recursos, el talento 
humano, el conocimiento, las actitudes y los comportamientos. Para Carbonell, (2002) la innovación educativa es un conjunto de ideas, procesos y estrategias, con las que se trata de iniciar e incentivar cambios en las practicas educativas. Por otro lado, para Llano la innovación educativa se vuelve en el tiempo una educación disruptiva puesto que ésta tiene el potencial de impactar a todo el contexto educativo (Llano, 2015).

La educación disruptiva permite la introducción de avances e innovaciones en los procesos educativos a través de las nuevas tecnologías y los nuevos usos que se abren en el ámbito comunicativo. Por tanto, para llevar a cabo un proceso de educación disruptiva en el aula, la labor docente se centra en propiciar espacios que generen nuevas experiencias a los estudiantes. Es necesario tener claro que los actores del proceso enseńanza y aprendizaje deben estar abiertos constantemente a transformar los espacios, los horarios, la metodología y, en definitiva, la forma de organizar el aprendizaje en clase, para permitir transformaciones importantes y disruptivas que amplíen los conocimientos y se fomente el aprendizaje significativo (Reveles, 2018).

Al hablar de un aprendizaje significativo, se hace referencia a una visión más allá de lo tradicional, en la cual la educación sea innovadora y creativa; ya que los estudiantes aprenden de una manera dinámica, no solo con los materiales comunes, sino con las Tecnologías de la Información y Comunicación (TICs). En la actualidad el uso de las TICs dentro del aula está tomando mayor importancia, los nińos y jóvenes tienen acceso a ella con mayor facilidad y las habilidades de uso son casi innatas. Incluir los recursos tecnológicos en el salón de clase requiere de una presencia docente para que los estudiantes tengan un aprendizaje significativo, mejoren su creatividad, se incremente la motivación hacia el estudio y la clase se vuelva más dinámica y colaborativa.

Lograr una adecuada presencia docente en ambientes de aprendizaje colaborativo mediados por TIC exige apropiarse de los nuevos roles adscritos a ella; teniendo presente que hay distintas etapas de planeación, construcción de recursos, administración e interacción y moderación con quienes participan (Bautista, Martínez \&
Hiracheta, 2014), (Rojas-Montero \& DíazBetter, 2018). Siendo una de las estrategias que el maestro utiliza en clase para enseñar, es despertar la curiosidad del estudiante; a través de nuevas estrategias didácticas y recursos educativos, como la tecnología. Parece entonces oportuno, mezclar los tres elementos para hacer del aula de clase un espacio interactivo cargado de creatividad y que deje huella en los estudiantes. Estos elementos son la educación, la tecnología y la innovación, que guiados a través de novedosas metodologías se generen estrategias para desarrollar la creatividad de los maestros (González, 2015).

El presente trabajo es una secuencia de un estudio realizado anteriormente en el que se aplicó la Metodología de Aprendizaje Basada en Metáforas Narrativas y Gamificación a 62 maestros de educación primaria, secundaria y superior que se están formando en un programa de Maestría de Educación, Innovación y Liderazgo en una universidad de Quito, Ecuador. El objetivo de esta metodología, fue generar interacción en jornadas largas de clase, mediante la aplicación de estrategias didácticas lúdicas para generar interacción y motivación. A través de una plataforma Moodle se diseñó un escenario gamificado, usando la metáfora de la Academia de Magia Hogwartz para resolver retos, con el fin de conocer nuevas herramientas tecnológicas que pueden aplicarse en el aula. En ese estudio los participantes tenían el rol de estudiantes, es decir usaban las herramientas como usuarios finales y no como administradores y diseñadores de las interfaces y contenidos (Jadán-Guerrero \& Ramos-Galarza, 2018). Siguiendo desde esta línea, el objetivo de este nuevo estudio es el de introducir el uso de tecnologías disruptivas en el aula mediante estrategias lúdicas y pensamiento de diseño para su aplicación didáctica. Para ello se aplican las metodologías Design Thinking y Game Thinking, que propician un ambiente de generación de ideas y prototipado rápido mediante actividades lúdicas. Se profundiza el conocimiento en aplicaciones de evaluación en clase, como Kahoot, Quizizz, Plickers y Google Forms. Los participantes aprenden a diseñar cuestionarios y aplican estos a sus estudiantes en un escenario real. Por otro lado, aprenden a generar códigos QR (Quick Response Barcode) y usar etiquetas NFC (Near Field Connection) con el fin de integrar dispositivos móviles a recursos 
tradicionales del aula. Aprenden Realidad Aumentada a través de aplicaciones, como Animal 4D +, Space 4D +, Humanoid 4D + y QuiverVision 3D Augmented Reality. Con las cuales desarrollan cuentos interactivos o juegos de mesa inclusivos. Finalmente, aprenden a usar sensores RFID (Radio Frequency IDentification) y la tarjeta electrónica Makey Makey con la plataforma de programación Scratch para diseñar recursos educativos tangibles, que combinan la información digital con materiales tradicionales del aula, tales como cartulina, colores, papeles brillantes, papel aluminio, plastilina, frutas o agua.

\section{Tendencias en pedagogía y tecnología}

En los últimos años, la presencia de la tecnología está cambiando la manera de enseñar y; una nueva generación de tecnologías disruptivas está ganando popularidad en las aulas. Ya es común ver pizarras digitales, que son superficies aumentadas por computadora con interacción multitáctil; así como tabletas y dispositivos móviles (Martínez, 2017). Este cambio tecnológico está propiciando el desarrollo de nuevas tendencias pedagógicas que van creciendo y no da señales de detenerse, sino que su avance se incrementará con el pasar del tiempo (Adell \& Castañeda, 2012).

Un reporte realizado por el Instituto Tecnológico de Monterrey en el año 2017 presenta un radar de innovación educativa, cuyo objetivo fue determinar las tendencias pedagógicas y las tendencias tecnológicas más relevantes desde la perspectiva de profesores innovadores de la institución. Los resultados mostraron que las tendencias en Pedagogía más utilizadas son: el aprendizaje activo, aprendizaje basado en problemas, aprendizaje basado en proyectos, aprendizaje colaborativo, aprendizaje basado en desafíos, aula invertida, creadores de espacio y gamificación. Por otro lado, las tendencias en tecnología son el aprendizaje adaptativo, aprendizaje en redes sociales y entornos colaborativos, aprendizaje móvil, aprendizaje ubicuo, aprendizaje con asistente virtual, MOOC (Massive Online Open Courses), Big Data y análisis de aprendizaje, laboratorios remotos y virtuales, Internet de las cosas, realidad aumentada y realidad virtual (EduTrends, 2017).
Estas nuevas tendencias, que han surgido en los últimos años, prometen fomentar nuevas formas de enseñanza y, la intención de ellas es que los centros educativos preparen a los estudiantes para un nuevo tipo de sociedad, la sociedad de la información, no solo enseñándoles a usar las TICs, sino también usándolas como herramientas de aprendizaje para enfrentar los nuevos desafíos educativos. Sin embargo, en Ecuador dentro del sistema educativo, el empleo de estas tecnologías se encuentra fragmentado, ya que no todas las instituciones educativas cuentan con los elementos esenciales y necesarios para su correcta aplicación. El uso de las tecnologías no es el adecuado, debido a la falta de capacitación y actualización en este ámbito, o la falta de tiempo para explorar y diseñar nuevas estrategias didácticas, por ende, no se genera una interacción entre docente, estudiante y contenido curricular.

Reconocer la importancia de las tendencias pedagógicas y tecnológicas, es trascendental en la educación actual, ya que es un desafío por su cambio constante, permitiendo a niños y jóvenes a desarrollar habilidades para aprender, desaprender y reaprender durante el transcurso de su vida. En los estudiantes se requiere que se despierte la motivación, que se fomente el trabajo colaborativo. A nivel de las instituciones educativas ecuatorianas, se deben incluir tecnologías educativas que integren el trabajo cotidiano de la institución, contribuyendo al desarrollo de las competencias necesarias en la formación de los futuros ciudadanos, de igual manera es urgente la capacitación de los docentes.

Hoy, no se trata de utilizar las tecnologías digitales para hacer "más" dinámica la clase, replicando prácticas que podrían realizarse sin estas tecnologías, sino que se trata de mirar lo que los estudiantes hacen, cómo se comunican, lo que comparten y crean, para poder recuperar y dar la importancia de acuerdo a la intencionalidad pedagógica a través de propuestas que los ayuden a seguir aprendiendo. En este estudio, se seleccionaron tres tendencias pedagógicas (aprendizaje colaborativo, aprendizaje basado en retos y gamificación) y cuatro tendencias tecnológicas (b-learning, m-learning, realidad aumentada y espacio makers). Aplicando la metodología Design Thinking se identifican necesidades y se abre un espacio de creatividad e innovación. Mientras que con la metodología Game 
Thinking se busca un ambiente competitivo, en el que los participantes mediante actividades lúdicas despierten su interés y motivación al uso de la tecnología. Esta combinación de metodologías facilita la introducción de herramientas tecnológicas que primeramente son evaluadas como usuarios finales y posteriormente como usuarios administradores, con la meta de que los participantes diseñen nuevos recursos educativos y no sólo se queden como prototipos, sino que sean evaluados en un escenario real con sus estudiantes.

\section{Metodología Design Thinking y Game Thin- king como recurso educativo}

La metodología Design Thinking se define como un proceso analítico y creativo que involucra a una persona en oportunidades para la generación de ideas innovadoras y que toma como centro la perspectiva de los usuarios finales para experimentar, modelar y crear prototipos, recopilar comentarios y rediseñar. De esta forma se pueden detectar problemas y necesidades, así como ofrecer soluciones efectivas y en muchos casos, alternativas, para cada una de ellas (Razzouk \& Shute, 2012; González, 2015). La metodología tiene cinco fases, como se muestra en la Figura 1.

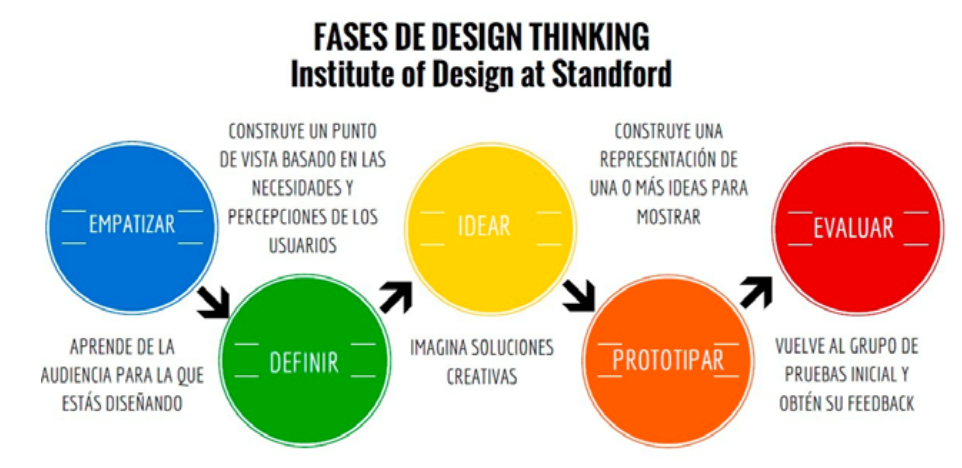

\section{Figura 1}

Fases de la metodología Design Thinking (Insituto de Diseño de Standford)

Elaboración propia (2019)

La fase Empatízar comienza con empatía, con un enfoque humano profundo, para obtener información de la audiencia, conocer sus necesidades y revelar nuevas formas de ver o identificar problemáticas inexploradas. La fase Definir, implica replantear el problema o desafío percibido y obtener perspectivas, lo que permite una visión más integral del camino hacia un punto de vista basado en las necesidades y percepciones de los usuarios, en este caso de los estudiantes. En la fase Idear, se fomenta el trabajo en equipo colaborativo y multidisciplinario para aprovechar las habilidades, personalidades y estilos de pensamiento de los participantes para resolver problemas multifacéticos. En la fase Prototipar se crea un espacio abierto para plasmar las ideas y se construya un prototipo de la solución. En la fase Evaluar se prueba el prototipo en un escenario real. La metodología Game Thinking o pensamiento del juego introduce aproximaciones creativas provenientes de los juegos para convertir actividades a priori complejas en dinámicas que motiven a las personas, invitando a una cooperación lúdica orientada a la innovación. El pensamiento del juego está comenzando a aparecer en una amplia variedad de contextos que no son juegos. El pensamiento del juego se ha descrito como un término general que abarca la gamificación, los juegos serios, el diseño inspirado en el juego y el juego que se puede utilizar para resolver algún tipo de problema. El proceso de usar el pensamiento de juego, consiste en aplicar mecánicas y dinámicas de los juegos para atraer a los usuarios y resolver problemas (Marczewski, 2015; De Puy \& Miguelena, 2017).

Existen algunos estudios que utilizan esta metodología en el ámbito educativo, entre ellas, una llevada a cabo en la Universidad de La Laguna en España en la que desarrolla estrategias para trabajar la creatividad en la Educación Superior. En este trabajo, se describe una experiencia de innovación educativa en la enseñanza de la Ingeniería y el Diseño, en donde los autores combinan varias estrategias de enseñanza, el aprendizaje basado en proyectos (PBL) y el aprendizaje basado en juegos, así como las técnicas de pensamiento de diseño (Design Thinking), pensamiento visual (Visual Thinking), pensamiento de juegos (Game Thinking) al proceso de enseñanza-aprendizaje. De los resultados encontrados se observó que el trabajo en equipo es una de las cuestiones más importantes que se logró con este tipo de metodologías híbridas. Asimismo, el trabajo en proyectos reales con metodologías de innovación y creatividad, favorecieron la implicación y motivación de los estudiantes en los mismos (González, 2015). 


\section{Materiales y Métodos}

\section{Participantes}

El programa de Maestría en Educación, Innovación y Liderazgo de la universidad en la que se llevó a cabo este estudio cuenta con aproximadamente 600 maestrantes, divididos en el $50 \%$ en la sede Ambato y 50\% la Sede Quito, que corresponde al universo del estudio. Para la muestra se tomaron dos paralelos del módulo de Infopedagogía Educativa, el $51 \%$ de la muestra fue seleccionada de la Sede Ambato y el 49\% restante de la Sede Quito y fue de tipo no probabilística ya que se trabajó con cuatro grupos intactos, siendo un total de 120 maestrantes. Las edades de los maestrantes estaban comprendidas entre 25 y 45 años, de los cuales el $82 \%$ son mujeres y el $18 \%$ varones. En cuanto al nivel de docencia que imparten los maestros de la muestra, se tiene que el $75 \%$ se concentra en la educación básica, seguida del $20 \%$ en la educación secundaria y el $5 \%$ en la educación superior. Los grupos de estudio están conformados por: Grupo 1 de 31 maestrantes, Grupo 2 de 31 estudiantes y Grupo 3 y 4 de 29 maestrantes. El primero y segundo en el período B18 correspondiente a julio-diciembre de 2018 en la sede matriz; mientras que el tercero y cuarto en el período A19 correspondiente a enero-junio de 2019 en la extensión.

\section{Instrumento}

El instrumento para la recolección de evidencias y narraciones fue la plataforma Moodle, en la cual se diseñaron estrategias de juego para desarrollar las actividades. En la Sede Ambato se utilizó la metáfora de la Academia de Magia de Hogwarts, mientras que en la Sede Quito la metáfora del Jurado que está juzgando la educación tradicional. En estos escenarios lúdicos se recogen fotos, videos y comentarios en foros de discusión. Esta información es procesada mediante análisis de contenido. Por otro lado, dentro de este estudio, también se aplicó la técnica de observación, que es un recurso muy útil para analizar el comportamiento de usuarios dependiendo del contexto del estudio. En este caso maestros de educación inicial, secundaria y superior (Hernández-Sampieri \& Mendoza, 2018). También se utilizaron las consultas para determinar la preferencia por determinada herramienta y su nivel de complejidad.

\section{Tipo y diseño}

El estudio es de tipo exploratorio-descriptivo, ya que aborda una temática poco estudiada, relacionada a la experiencia de maestros con tecnologías disruptivas. Asimismo, es de tipo descriptivo, ya que a través de la observación de los fenómenos en su ambiente natural se describen las experiencias innovadoras en el aula y el análisis del desarrollo de la creatividad, desde un punto de vista Psicológico (Ramos, 2019).

\section{Procedimiento}

La asignatura de Infopedagogía Educativa fue organizada siguiendo una modalidad b-learning, es decir un estilo de aprendizaje mediado por una plataforma tecnológica para realizar actividades en el aula y actividades autónomas, las cuales se distribuyeron a lo largo de 5 semanas, destinando 40 horas presenciales, 40 horas de tipo virtual-online y 20 horas al trabajo autónomo. Las actividades de enseñanza-aprendizaje se diseñaron bajo la metodología Game Thinking, es decir basadas en actividades lúdicas. Dichas actividades fueron planteadas en función de la metodología Design Thinking, como se describe a continuación en cada fase.

\section{Empatizar}

Con el fin de conocer a los participantes y comprender de manera más profunda sus problemas y expectativas, se organizó a los participantes en grupos, en el caso de la Academia de Magia Hogwarts los participantes debían inscribirse en una de las 6 casas de confraternidad: Ravenclaw; Hufflepuff; Slytherin; Gryffindor; Slytherpuff y Meilen. En el caso de la metáfora de Jurado, los participantes debían inscribirse en uno de los 6 Bufetes de Abogados: STEM (Science, Technology, Engineering \& Mathematics); Lenguaje y Literatura; Educación Especial e Inclusiva; Estrategias Educativas; Innovación y Emprendimiento; y Liderazgo Educativo. Con el fin de recabar no sólo datos y estadísticas de los participantes, sino de analizar situaciones, comprender su actividad 
académica, así como los diferentes problemas y necesidades que poseen se desarrollaron actividades lúdicas en el aula y en la plataforma, en el primer caso el "El muro de los lamentos" y en el segundo caso "Buscando pistas en la ciudad".

\section{Definir}

En esta fase fue esencial definir los problemas de los participantes según el área de desarrollo laboral y cuál de ellos es más relevante para plantear una solución. En este caso se diseñó un foro de discusión en la plataforma, en el cual los participantes fundamentan su problema mediante fuentes bibliográficas, para ello debían realizar una visita virtual a la "Biblioteca de Hogwarts" o "Biblioteca Cosrpus Lex", respectivamente.

\section{Idear}

Con el problema definido en cada grupo, en esta fase, empieza el proceso de generación de ideas, para ello el "Mago Mayor" o el "Juez" llama a una audiencia y somete a los participantes a un cuestionamiento de los problemas encontrados, para ello se utilizaron las palabras mágicas "Kahoot, Quizizz y Plickers" y la vartita mágica (dispositivo móvil) en el caso de la Academia Hogwarts y en el caso del Jurado los "Clickers (sencillo sistema de respuesta personal remota)" mediante el uso de dispositivos móviles. Kahoot!, Quizizz y son tres herramientas tecnológicas para evaluación, que representan una nueva generación de sistemas de respuesta basadas en un enfoque de la motivación a través de la gamificación (Inge Wang \& Lieberoth, 2016).

\section{Prototipar}

En esta fase, el propósito fue el de convertir la idea o solución en un prototipo digital o físico y esto lo hicieron en un trabajo colaborativo en cada grupo. Para el efecto, se planteó la modalidad Space Makers con los talleres "Taller de Magia" y "Armando el rompecabezas de pistas", respectivamente para cada metáfora narrativa. En estos talleres se utilizaron material tradicional del aula como: papel; cartón; colores; marcadores; acuarelas; plastilina y papel de aluminio, para dar vida a unos personajes mágicos o buscar pistar con la magia de la Realidad Aumentada que proveen las Apps Animal 4D +, Space 4D +, Humanoid 4D + y QuiverVision 3D Augmented Reality. Poste- riormente, se hacía una visita virtual a las "mascotas de Hogwarts" o se invitaba a "los testigos", metáforas de las plataformas web Scratch y MIT AppInventor, que hablan un lenguaje diferente y por ello los participantes debían aprender a programar por bloques e interpretar códigos $\mathrm{QR}$ escondidos. Los miembros de cada grupo debían usar algunas herramientas para presentar un prototipo, entre ellas la App Hi-Q MP3, para grabar sus narraciones o conjuros; así como sensores y etiquetas RFID (Radio Frequency Identification), etiquetas NFC (Near Field Connection) y la placa electrónica Makey-Makey para combinar recursos educativos tradicionales con recursos educativos digitales.

\section{Evaluar}

En esta fase se realizan pruebas de los prototipos realizados, primeramente, una evaluación interna entre los diferentes grupos. Con la retroalimentación de todos los participantes se plantea un reto "challenge" para que este prototipo sea evaluado en un escenario real. Para ello los participantes en el transcurso de una semana debían replicar la evaluación con sus estudiantes y recabar evidencias. Toda esta información debía ser subida en la plataforma Moodle; así como sus opiniones y comentarios de la experiencia.

\section{Resultados}

Los resultados obtenidos en el estudio se enfocan en el uso de las herramientas tecnológicas y el desarrollo de la creatividad para generar nuevos recursos educativos apoyados por la tecnología, en este contexto, los resultados se presentan en tres categorías: 1. Gamificación; 2. Recursos Educativos digitales; y 3. Recursos Educativos híbridos.

\section{Gamificación}

El resultado de la metodología Game Thinking fue muy alentador ya que los participantes descubrieron nuevas formas de aprender, mediante herramientas que ayudan a gamificar el aula. En el estudio se utilizaron Kahoot, Quizizz y Plickers. Gracias a los testimonios de los particpantes fue una experiencia muy enriquecedora. En las narra- 
tivas de los participantes se identificó que gracias a estas herramientas de evaluación y coevaluación disminuyeron considerablemente su ansiedad y miedos a la hora de enfrentarse a nuevos conocimientos. Cada una de las heramientas tiene su particularidad. Kahoot permite la creación de pruebas, debates, encuestas y recolocación de respuestas. En cualquiera de ellas el profesor proyecta un PIN que se genera automáticamente con la prueba seleccionada, se muestra una pregunta con la ayuda de un proyector, mientras que los estudiantes seleccionan una opción en una computadora o en un dispositivo móvil mediante figuras geométricas de colores que representan las opciones de respuesta. En la Figura 2 se muestra un ejemplo de una pregunta.

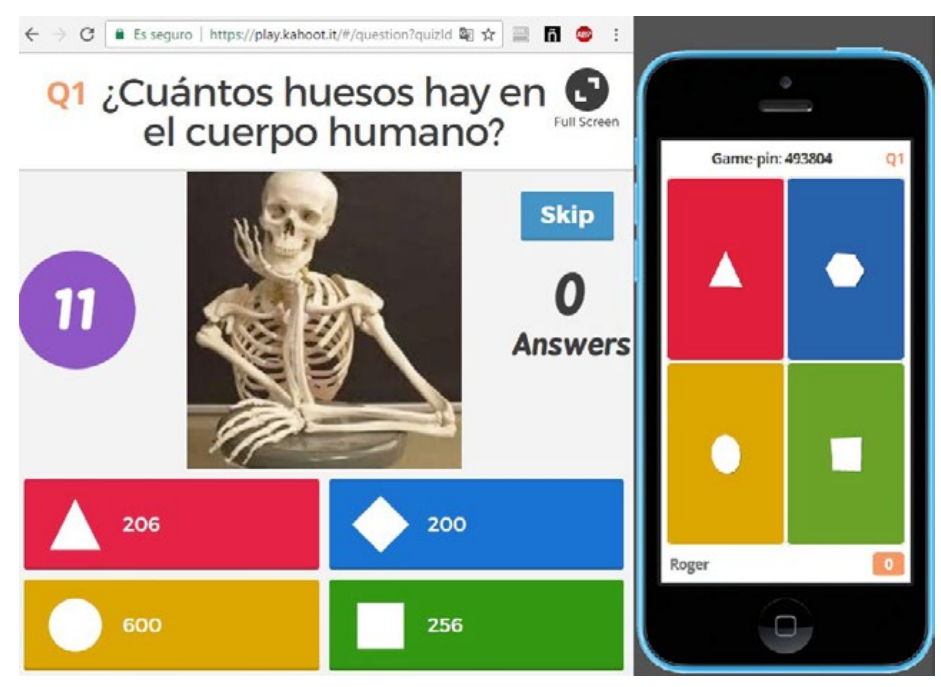

Figura 2

Kahoot muestra en el proyector la pregunta y el dispositivo móvil las opciones

Cuando termina la prueba, los estudiantes se ven reconocidos en un ranking de rapidez y aciertos, lo cual supone gratificación personal y una retroalimentación inmediata sobre sus resultados. De esta manera, son capaces de valorar su puntuación, aprender de sus errores, corregirlos y mejorarlos.

También se hizo uso de la herramienta Quizizz, que a diferencia de la anterior, las preguntas se muestran en la computadora o dispositivo móvil. En el proyector se muestra el progreso de cada estudiante, de color verde las correctas y de color rojo las incorrectas y va generando un ranking.
Los participantes fueron capacitados en el diseño de los cuestionarios para que posteriormente lo pongan en práctica en su aula. Los resultados fueron alentadores, ya que subieron a la plataforma evidencias y comentarios positivos en aspectos de participación y motivación. En la Figura 3 se muestra un ejemplo aplicado en una aula con Quizizz.

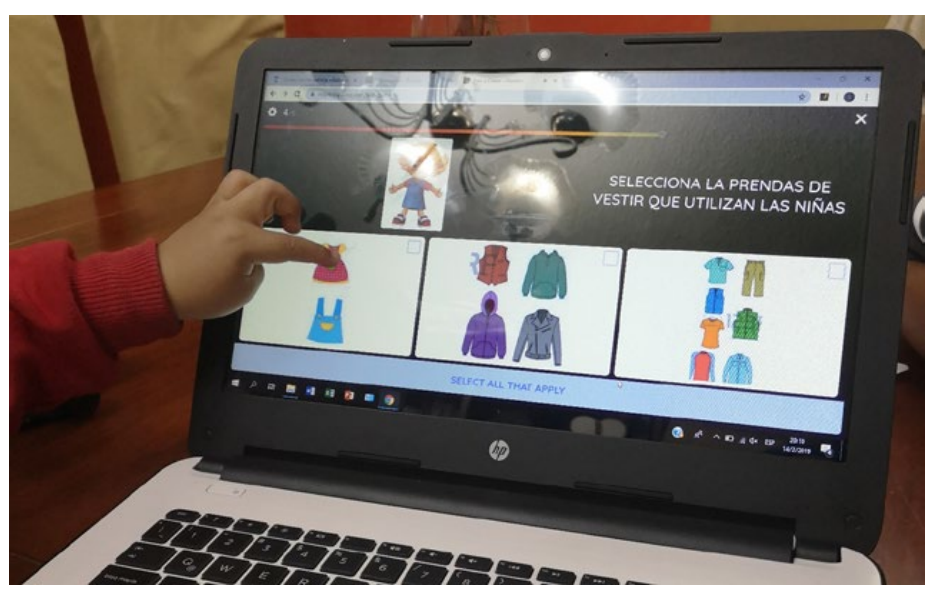

Figura 3

Uso de Quizizz por parte de participantes en una aula

Finalmente, se aplicó Plickers, una herramienta que a diferencia de las anteriores no necesita una computadora o dispositivo móvil para contestar a las preguntas, en su defecto, genera un código QR para cada participante o grupo ingresado en una clase. En Plickers, primero se crea una clase, posteriormente se da de alta a los estudiantes de la clase. Se crean conjuntos de preguntas y de ellos se pueden extraer una o varias para una clase determinada. La ventaja de esta herramienta es que no necesita que los estudiantes cuenten con un dispositivo móvil. En la aplicación se entregó una tarjeta impresa a cada casa de confraternidad o bufete de abogados. Cada código $\mathrm{QR}$ de la tarjeta tenía a cada lado una alternativa A-B-C-D. Después de proyectar una pregunta, el profesor con un dispositivo móvil conectado a Internet pudo leer las respuestas representadas en cada tarjeta. Los participantes ubicaron la tarjeta en una posición de acuerdo a su respuesta, mientras que el profesor escaneaba los resultados, que eran mostrados en pantalla. Esta herramienta resultó más compleja para los participantes porque la interfaz de diseño del cuestionario no fue tan intuitiva como las dos anteriores. Sin embargo, causó motivación y participación de los equipos. En la 
Figura 4 se muestra un ejemplo en un escenario real y se puede observar las expresiones de los participantes.

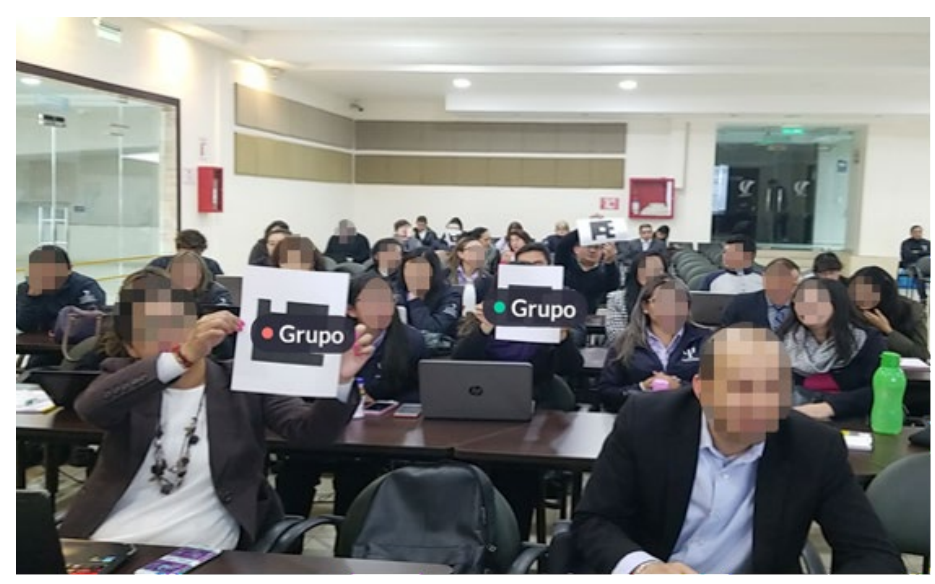

Figura 4

Docentes responden a una pregunta con un código QR en Plickers

En todas las aplicaciones se puso a prueba el número de participantes, logrando obtener hasta 31 usuarios conectados a la vez. En este caso Kahoot y Quizizz si mostraron problemas de conexión en un número mayor a 20 participantes. Ante este problema los maestros diseńaron una estrategia de grupos y dieron solución a este problema de conectividad. A pesar que Plickers no tenía este problema, los maestros prefirieron usar en su fase de evaluación las dos primeras. Estudios similares de gamificación en el aula coinciden en que estas herramientas generan colaboración, participación y motivación, además que permiten llevar un control instantáneo del progreso del alumno de manera individual y de la clase en conjunto (Dellos, 2015; López García, 2016).

Los resultados encontrados con el instrumento de consulta con respecto al uso de estas herramientas en sus aulas en la fase de evaluación, permitieron identificar que Kahoot y Quizizz fueron más intuitivas en el diseño de cuestionarios y más entretenidas a la hora de aplicar en el aula. Mientras que Plickers a pesar que no se requiere que todos los participantes tengan un dispositivo móvil, no fue ampliamente aplicada en la fase de evaluación, como se puede ver en la Figura 5.

Parece ser que el aprendizaje basado en juegos es una buena práctica en educación y encontrar formas de integrar juegos competitivos en el aula ¿que herramienta utilizó en su aula de clase?

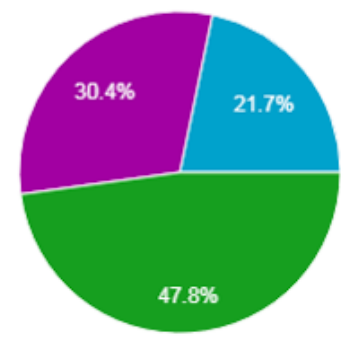

Kahoot!

Quizizz

Plickers

\section{Figura 5}

Estadísticas de uso de las herramientas de evaluación

que promuevan el aprendizaje es esencial para profesores y estudiantes.

Recursos educativos con realidad aumentada y códigos QR

En esta categoría, los equipos diseñaron recursos educativos que combinaron materiales de aula tradicionales con dispositivos móviles. Para ello usaron códigos QR y aplicaciones de realidad aumentada. La inclusión del software que lee códigos $\mathrm{QR}$ en dispositivos móviles permitió darle nuevos usos para compartir información visual dentro del ámbito educativo, pues permite conectar cualquier contenido a un código $\mathrm{QR}$, ya sea un URL o contenido multimedia. Siguiendo las fases de la metodología Design thinking los equipos desarrollaron su creatividad y crearon varios prototipos relacionados al problema que cada uno de ellos tenía en el equipo STEM se dieron cuenta que los estudiantes presentan falencias en el aprendizaje de las Matemáticas, para ello diseñaron el recurso educativo "Twister preguntón, que consistía en un conjunto de códigos QR relacionadas a preguntas de, si un participante contestaba erroneamente a una pregunta, tenía que ejecutar un reto en el tablero del juego. En la figura 7 se muestra la demostración del juego.

Para este tipo de aplicaciones se capacitó a los equipos a programar en MIT App Inventor, en el cual se integraron imágenes y audios en formato MP3 grabados por los mismos participantes con la aplicación Hi-Q MP3. Esta actividad propició el trabajo en equipo y según los comentarios de los particpantes, les permitió descubir nuevos ele- 
despertar el interés de los estudiantes en el proceso de enseńanza-aprendizaje (Monedero et al., 2017). Por otro lado, la realidad aumentada agrupa aquellas tecnologías que permiten la superposición, en tiempo real, de imágenes, marcadores o información generados virtualmente, sobre imágenes del mundo físico y generan gran interés en los estudiantes (De la Horra Villacé, 2016).

\section{Prototipos de Recursos Educativos híbridos}

Para los recursos educativos híbridos, los equipos fueron capacitados en el uso de Scratch y Makey Makey. Scratch, que es una plataforma diseñada para desarrollar habilidades de pensamiento lógico a través de un lenguaje de programación sencilla, el mismo que en su aplicación se puede interactuar de manera didáctica por medio de piezas (bloques) de rompecabezas. Este conjunto de instrucciones permiten desarrollar la creatividad para escribir historias y juegos interactivos. Makey Makey es una placa electrónica que extiende las funciones del teclado y ratón de la computadora, lo que permite enviar órdenes al computador al que se encuentre conectado por medio de un cable USB. En vez de pulsar las teclas de las flechas o barra espaciadora lo que hace es cerrar el circuito mediante contactos o pinzas de cocodrilo $\mathrm{y}$ de esta forma se simula haber pulsado una tecla. Lo cual permite convertir cualquier objeto de la vida diaria en un teclado, un mando o un ratón al brindar a los usuarios conectar objetos cotidianos a programas de computadora o bien programarlos con Scratch (Strawhacker et al., 2015).

Uno de los prototipos híbridos considera el aprendizaje de la lateralidad con la música y el baile. La figura 9 muestra la interfaz híbrida con cartulina y papel de aluminio.

En el área de lenguaje y literatura, los maestros diseñaron un prototipo construido con Scratch y Makey Makey para fortalecer la conciencia fonológica y la asociación. Para ello crearon un cuento digital, en el cual un estudiante después de escucharlo crea elementos o personajes del cuento con plastilina. Estos elementos se conectan a la computadora a través de Makey Makey y Scratch. La estrategia didáctica fue la de asociación de los elementos digitales con los físicos basados en la

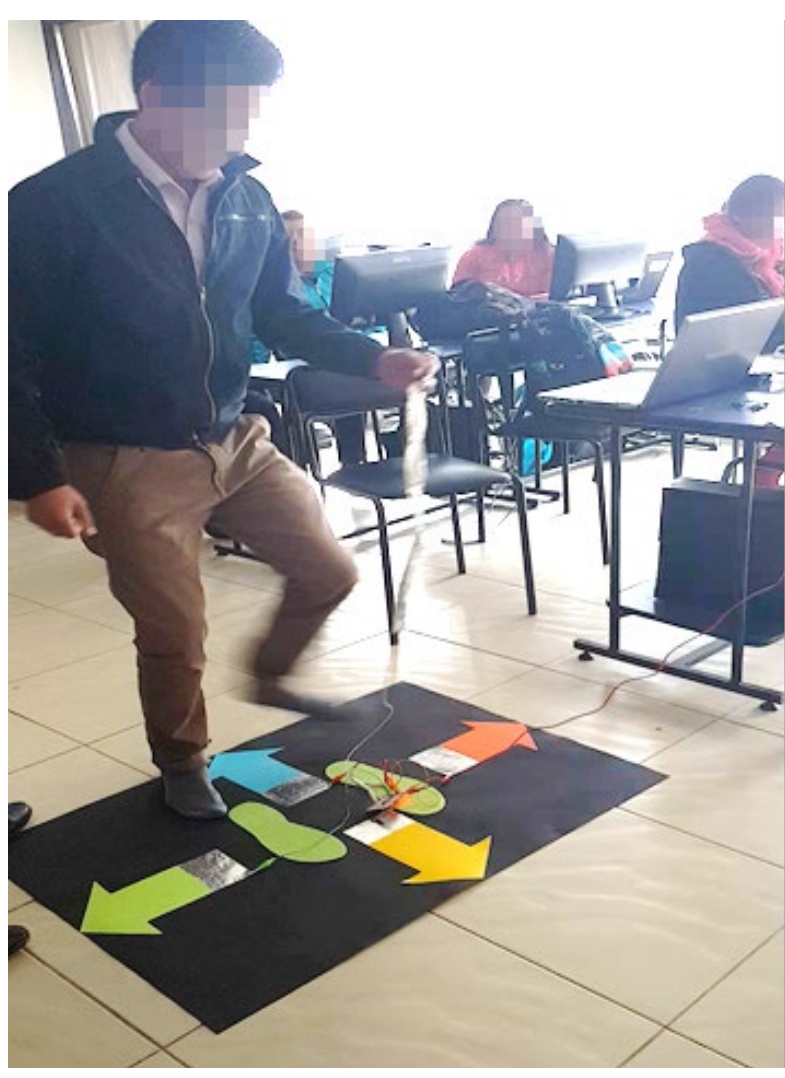

Figura 9

Tablero dance, dance revolution construído con cartulina y papel aluminio

relación pictograma-fonema Esta propuesta esta orientada a niños con necesidades educativas especiales para mejorar su nivel de motricidad fina y lectura. Según un estudio que se hizo con interfaces tangibles, estas favorecen a la estimulación sensorial y al aprenizaje significativo (Fan \& et.al, 2017). En la Figura 10 se muestra el prototipo de conciencia fonológica.

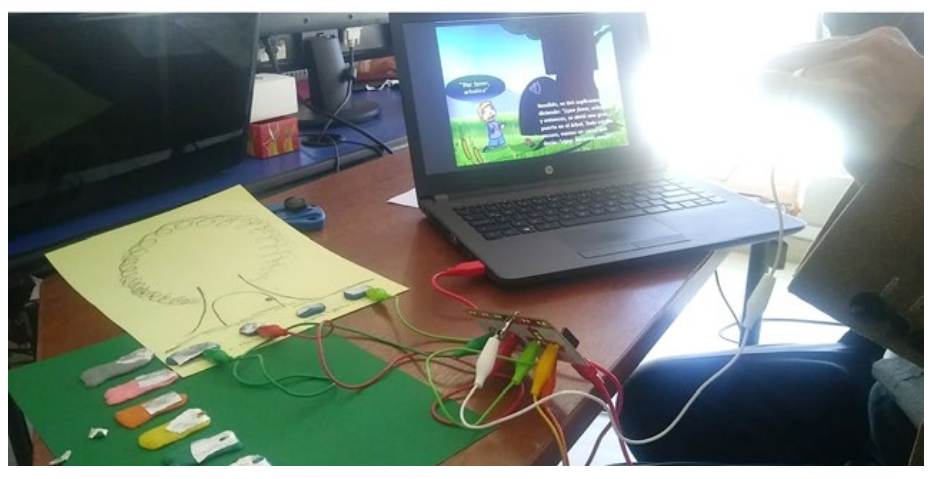

Figura 10

Prototipo de conciencia fonológica construído con cartulina y plastilina

En el área de las ciencias naturales, un grupo diseñó una placa similar a la anterior, pero en lugar 
de letras tenía los órganos del cuerpo humano hechos de plastilina. Explican los maestros que sus estudiantes pueden construir los órganos del cuerpo humano y mediante Makey Makey y un programa en Scratch pueden mostrar información adicional. En la Figura 11 se muestra el tablero del cuerpo humano.

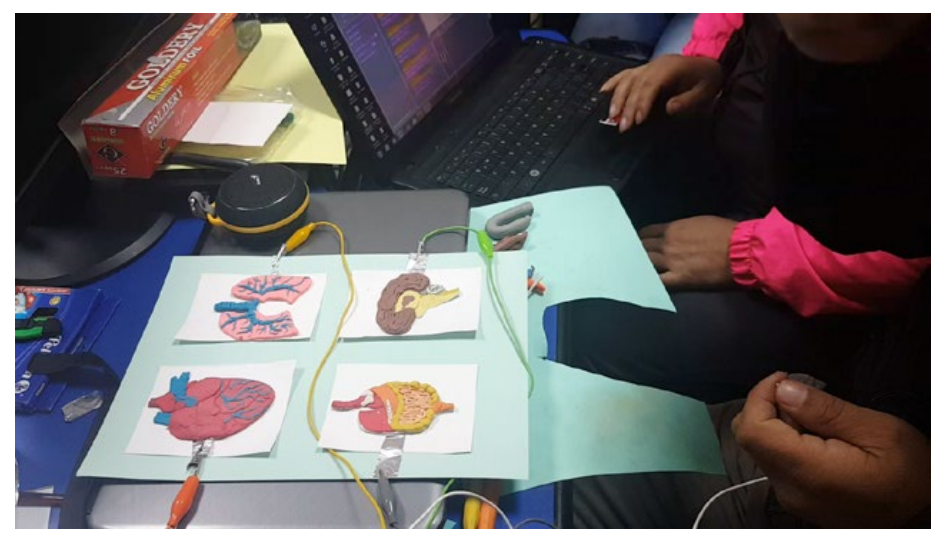

Figura 11

Tablero de órganos del cuerpo humano construído con cartulina y plastilina

\section{Discusión y Conclusiones}

Uno de los problemas a los que se enfrentan los maestros en el aula, es la forma de evaluar los objetivos propuestos en la malla curricular y la forma de controlar el proceso de asimilación de los aprendizajes, así como el grado de dominio de los contenidos por parte de los estudiantes. La tecnología ha permitido poder hacer este proceso de manera inmediata y rápida; sin recurrir a los controles habituales que presionan al alumnado, y siendo copartícipe de estos objetivos el estudiantado; estimulándolos de una forma lúdica y gráfica (Educación 3.0, 2015).

En este trabajo se ha presentado una experiencia de innovación educativa siguiendo una metodología Design Thinking y Game thinking que intenta potenciar la creatividad e innovación de 120 maestros de educación primaria, secundaria y superior a través de un pensamiento lúdico. Para desarrollar esta experiencia se ha seguido la técnica b-learning por medio de una plataforma Moodle, en la cual se planifican las actividades grupales e individuales y se recogen las evidencias y testimonios de los maestros. Asimismo, se trabajó para desarrollar las competencias transversales de los maestros a través del aprendizaje significativo, las actividades se centraron en los problemas mencionados en la fase de empatía. Se ha podido observar que el $100 \%$ de los participantes dispone de un teléfono inteligente, sin embargo, su utilización en el ámbito académico es escaso, pero si se destaca el uso de Whatsapp como herramienta de comunicación y coordinación grupal. De los resultados cualitativos se observó que el trabajo en equipo fue una de las características más importantes en el aprendizaje basado en retos, que permitió el trabajo en proyectos reales con metodologías de innovación y creatividad.

Las estrategias para desarrollar la creatividad y la innovación a través del pensamiento de diseño y aprendizaje basado en retos y juegos permitieron generar prototipos de recursos educativos apoyados por la tecnología. Se observó que la experiencia desarrollada fue muy gratificante, ya que observó a los participantes altamente motivados y desarrollaron prototipos creativos e innovadores, se logró una alta participación en las sesiones presenciales y en las actividades virtuales. Aunque los resultados fueron positivos, es necesario mencionar algunas de las limitaciones que se presentaron en este estudio, una de ellas era la incomodidad que sintieron algunos de los participantes al saber que usarían la tecnología, de antemo existía esa preconcepción de que èsta es compleja y difícil de usar, sin embargo al utilizar la metodología Game Thinking pudieron evidenciar con el juego, que no fue tan compleja como pensaban y fueron capaces de usarla. De igual manera, pasó cuando sabían que serían evaluados o tenían que aprender a programar, tal vez porque se imaginaban evaluaciones tradicionales o lenguajes de programación complejos. Cuando pudieron ver los resulatdos obtenidos reflexionaron que existen herramientas fáciles de usar, como Kahoot, Quizizz y Plickers para evaluación; así como Scratch y MIT App Inventor para programar actividades sin saber programar. Otra limitación fue la capacidad de memoria y la diversidad de los dispositivos móviles de los participantes, que hizo que se desvíe el desarrollo de las estrategias didactica a la limpieza y depuración de los dispositivos. 
Estas vivencias nos anima a continuar trabajando para replicar esta experiencia innovadora en futuras generaciones. Como trabajo futuro se va a profundizar en el desarrollo del prototipo del juego de mesa inclusivo, el cual creemos que tiene un gran potencial para integrar varias de las tecnologías descritas en el estudio, y además que contribuya a la introducción de la tecnología en el aula.

\section{Agradecimientos}

Un agradecimiento especial a los alumnos de la Maestría en Educación, Mención Innovación y Liderazgo Educativo (MEILE-3), por compartir sus vivencias en el módulo de Infopedagogía Educativa.

\section{REFERENCIAS BIBLIOGRÁFICAS}

Adell J. \& Castañeda L. (2012). Tecnologías emergentes, ¿pedagogías emergentes? Asociación Espiral, Educación y Tecnología, pp. 13-32

Arroyo, N., Campos, F., Carballo, M., González, G., Solano, A., Vargas, E., \& Carpio, M. (2019). El Modelo dual de reconocimiento de la palabra en el Sistema Braille. Revista CienciAmérica, 8(1), 90-104. https://doi.org/10.33210/ca. v8i1.194

Bautista M., Martínez A. \& Hiracheta R. (2014). El uso de material didáctico y las tecnologías de información y comunicación (TIC's) para mejorar el alcance académico, Ciencia y Tecnología, 14, 2014, 183-194.

Carbonell, J. (2002). La aventura de innovar. El Cambio en la Escuela, Ediciones Morata

De la Horra Villacé, I. (2016). Realidad aumentada, una revolución educativa. EDMETIC, 6(1), 9-22. https://doi. org/10.21071/edmetic.v6i1.5762

De Puy M. \& Miguelena R. (2017). Importancia de la Gamificación en la Educación Aplicado en Entornos de la Investigación, 15th LACCEI International Multi-Conference for Engineering, Education, and Technology: "Global Partnerships for Development and Engineering Education”, 1921 July 2017, Boca Raton Fl, United States.

Dellos, R. (2015). Kahoot! A digital game resource for learning, International Journal of Instructional Technology and Distance Learning, 12. (4).

Educación 3.0 (2015). Aprendizaje activo con sistemas de respuesta en Infantil y Primaria, Recuperado de https:// www.educaciontrespuntocero.com/experiencias/aprendizaje-activo-infantil-primaria/26144.html.
EduTrends (2017). Observatorio de Innovación Educativa, Reporte Edu Trends. Monterrey, México: Tecnológico de Monterrey

Fan, M., Antle, A. N., Hoskyn, M., Neustaedter, C. \& Cramer, E. S. (2017) Why tangibility matters: A design case study of at-risk children learning to read and spell. Proceedings of the 2017 CHI Conference on Human Factors in Computing Systems, USA, içinde (ss. 1805-1816). https:// doi.org/10.1145/3025453.3026048

González, C. (2015). Estrategias para trabajar la creatividad en la Educación Superior: pensamiento de diseño, aprendizaje basado en juegos y en proyectos. Revista de Educación a Distancia (RED), 40(2), 2-15. Recuperado de: http://www. um.es/ead/red/40

Hernández-Sampieri R. \& Mendoza, C. (2018). Metodología de la Investigación: Las rutas cuantitativa, cualitativa y mixta, Mc Graw Hill. https://doi.org/10.17993/CcyLl.2018.15

Inge Wang A. \& Lieberoth A. (2016). The effect of points and audio on concentration, engagement, enjoyment, learning, motivation, and classroom dynamics using Kahoot!, Academic Conferences and Publishing International Limited

Jadán-Guerrero, J. \& Ramos-Galarza, C. (2018) Metodología de Aprendizaje Basada en Metáforas Narrativas y Gamificación: Un caso de estudio en un Programa de Posgrado Semipresencial. Hamut'ay, 5 (1), 84-104. https://doi. org/10.21503/hamu.v5i1.1560

Llano, P. (2015). La innovación no es solo cambio de tecnología, AGLALA ISNN 2215-7360; 6 (1): 38-72. https:// doi.org/10.22519/22157360.909

López, N.J. (2016). Evaluación y TIC en Primaria: el uso de Plickers para evaluar habilidades musicales. ENSAYOS, Revista de la Facultad de Educación de Albacete, 31(2). Recuperado de http://www.revista.uclm.es/index.php/ensayos

Marczewski, A. (2015). User Types. In Even Ninja Monkeys Like to Play: Gamification, Game Thinking and Motivational Design. Recuperado de http://www.gamified.uk/usertypes/

Martínez, G. (2017). Tecnologías y nuevas tendencias en educación: aprender jugando. El caso de Kahoot. Revista Opción, 33 (83), 252-277.

Monedero C., Castro A. \& Tomás J. (2017). Videotutoriales y códigos QR: recursos TIC en laboratorios de Ciencias de la Comunicación, INNOEDUCA. International Journal of Technology and Educational Innovation, 3 (2), 137 - 145. https://doi.org/10.24310/innoeduca.2017.v3i2.2046

Navas, E., \& Caisachana, A. (2018). Juego Interactivo para Incrementar la Atención y Retentiva Visual en Nińos con Síndrome de Down. Revista CienciAmérica, 7(1), 30-36. Recuperado de http://cienciamerica.uti.edu.ec/openjournal/index.php/uti/article/view/149

Ramos, C. (2019). Fundamentos de investigación para psicólogos: primer round. Quito, Ecuador: Universidad Tecnológica Indoamérica. 
Razzouk, R., \& Shute, V. (2012). What Is Design Thinking and Why Is It Important? Review of Educational Research, 82(3), 330-348. https://doi. org/10.3102/0034654312457429

Reveles J. (2018). La educación disruptiva transforma el aprendizaje, El sol de Zacatecas. Recuperado de https:// www.elsoldezacatecas.com.mx/analisis/la-educacion-disruptiva-transforma-el-aprendizaje-1156622.html

Rojas-Montero, J. \& Díaz-Better, S. (2018) Presencia Docente en Ambientes de Aprendizaje Mediados por Tecnologías de la Información y la Comunicación. Hamut'ay, 5 (1), 53-65. https://doi.org/10.21503/hamu.v5i1.1520

Strawhacker A., Lee M., Caine C. \& Bers, M. U. (2015). ScratchJr Demo: A coding language for kindergarten. Proceedings of the 14th International Conference on Interaction Design and Children (IDC '15). Boston, MA: ACM. https://doi.org/10.1145/2771839.2771867 